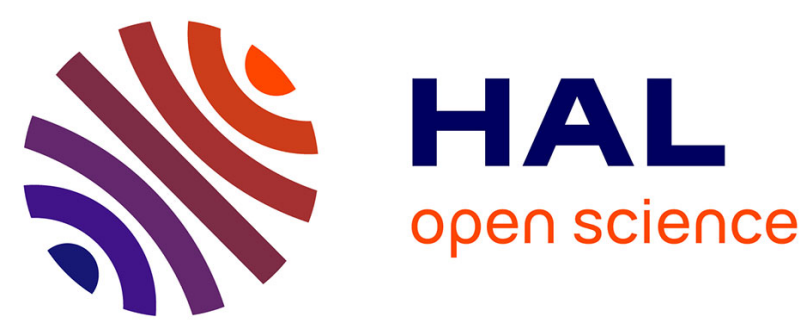

\title{
Sunlab: a Functional-Structural Model for Genotypic and Phenotypic Characterization of the Sunflower Crop
}

Fenni Kang, Paul-Henry Cournede, Jeremie Lecoeur, Véronique Letort

\section{To cite this version:}

Fenni Kang, Paul-Henry Cournede, Jeremie Lecoeur, Véronique Letort. Sunlab: a FunctionalStructural Model for Genotypic and Phenotypic Characterization of the Sunflower Crop. Ecological Modelling, 2014, 290, pp.21-33. 10.1016/j.ecolmodel.2014.02.006 . hal-01272125

\section{HAL Id: hal-01272125 \\ https://hal.science/hal-01272125}

Submitted on 10 Feb 2016

HAL is a multi-disciplinary open access archive for the deposit and dissemination of scientific research documents, whether they are published or not. The documents may come from teaching and research institutions in France or abroad, or from public or private research centers.
L'archive ouverte pluridisciplinaire HAL, est destinée au dépôt et à la diffusion de documents scientifiques de niveau recherche, publiés ou non, émanant des établissements d'enseignement et de recherche français ou étrangers, des laboratoires publics ou privés. 


\title{
Sunlab: a Functional-Structural Model for Genotypic and Phenotypic Characterization of the Sunflower Crop
}

\author{
Fenni Kang ${ }^{\mathrm{a}}$, Paul-Henry Cournede ${ }^{\mathrm{a}}$, Jeremie Lecoeur ${ }^{\mathrm{b}}$, Veronique \\ Letort $^{\mathrm{a}, *}$ \\ ${ }^{a}$ Laboratory MAS, Ecole Centrale Paris, Grande Voie des Vignes, F-92 295 \\ Chatenay-Malabry, France \\ ${ }^{b}$ Montpellier SupAgro, 2, Place Pierre Viala, 34060 Montpellier Cedex 2
}

\section{Abstract}

A new functional-structural model SUNLAB for the crop sunflower (Helianthus annuus L.) was developed. It is dedicated to simulate the sunflower organogenesis, morphogenesis, biomass accumulation and biomass partitioning to organs. It is adapted to model phenotypic responses of different genotypic variants to diverse environmental factors including temperature stress and water deficit. A sensitivity analysis was conducted to quantify the relative parameter influences on the main trait of interest, the grain yield. The model was calibrated for four genotypes on two experimental datasets collected on plants grown under standard non-limiting conditions and moderate water stress. Its predictive ability was then tested on an additional dataset. The four considered genotypes - "Albena", "Melody", "Heliasol" and "Prodisol" - are the products of more than 30 years of breeding effort. Comparing the values found for the four parameter sets associated to each variant, allows to identify genotype-specific parameters. The model also provides a novel way of investigating genotype performances under different environmental conditions. These promising results are a first step towards

\footnotetext{
*Corresponding author Tel.;(33) 141131765

Preprint submitted to Ecological did adelling com (Fenni Kang),

January 4, 2014 venonique.letort@ecp.fr (Veronique Letort)
} 
the potential use of the model as a support tool to design sunflower ideotypes adapted to the current worldwide ecological and economical challenges and to assist the breeding procedure.

9 Keywords: SUNLAB, SUNFLO, GREENLAB, Sunflower model

\section{Introduction}

As one of the major oilseed crops worldwide, sunflower production has to face the growing social demand in a context of strong ecological and economical constraints: growers are confronted to the challenge of increasing sunflower productivity under changing climatic conditions while maintaining low input levels and reduced costs. A partial response to this challenge could be found by breeding new genotypes or by identifying the best genotype, among a set of existing ones, for a given location and for given management practices; see for instance Allinne et al. (2009).

Assessments of genotype performances for in situ experimental trials hamper the breeding process by temporal, logistic and economical difficulties. Indeed, genotypes perform differently depending on the environmental conditions (soil, climate, etc.) and the management practices (sowing date, nitrogen inputs, irrigation, etc.). Therefore a large number of trials are needed to explore a sufficiently diverse set of genotypes $\mathrm{x}$ environment $\mathrm{x}$ management (GxExM) combinations in order to characterize these complex interactions. An emerging approach to overcome these difficulties relies on the use of models represented as a set of biophysical functions that determine the plant phenotype in response to environmental inputs. Models can help in breeding strategies and management by dissecting physiological traits 
into their constitutive components and thus allow shifting from highly integrated traits to more gene-related traits that should reveal more stable under varying environmental conditions (Yin et al., 2004; Hammer et al., 2006).

Consequently, an important question to examine is how to design models that can be used in that context. The models should simulate the phenotypic traits of interest (e.g. yield) with good robustness and predictive capacity. The models should also present a trade-off between mechanistic aspect and complexity: Chapman et al. (2003) state that, for such use, a growth model should include 'principles of response and feedbacks' to 'handle perturbations to any process and self-correct, as do plants under hormonal control when growing in the field' and to 'express complex behavior even given simple operational rules at a functional crop physiological level'. Casadebaig et al. (2011) discuss that question in the case of their model SUNFLO (Lecoeur et al., 2011). SUNFLO is a biophysical plant model that describes organogenesis, morphogenesis, and metabolism of sunflower (Helianthus annuus L.). It has shown good performances to identify, quantify, and model phenotypic variability of sunflower at the individual level in response to the main abiotic stresses occurring at field level but also in the expression of genotypic variability (Casadebaig et al., 2011). The authors mixed mechanistic and statistical approaches to deal with highly integrative variables such as harvest index $(H I)$. This $H I$ factor is determined by a simple statistical relationship dependent on covariables previously simulated by the mechanistic part of the crop model throughout the growing season. Although this statistical solution and the large datasets used for its parameterization conferred good robustness to the prediction of $H I$ and thereby crop harvest, biomass parti- 
tioning to other plant organs and trophic competition between organs were not taken into account. Fenni: Here, in last review, the third reviewer posed a question "The idea of formalizing trophic competition between organs ( $\mathrm{p} 4$ $127-33)$ is at the core of this new model. You write that such a formalization should help representing feedback effects of biomass partitioning on "other processes" (p 4 l 13-15). What do you mean?", I simply deleted the sentence that saying "feedback effects of biomass partitioning on other processes cannot be taken into account", but I still tried to mention it in the discussion saying "The plants are considered only at the minimal level of organ compartments. PBM model ignores plant architecture and its plasticity. The lack of individual organ's simulation can influence the simulation of plant functioning. For example, PBMs models normally use the relative values of the sink strength of organs to simulate biomass partitioning. These sink values are assessed directly from experiments and the sources and sinks have no significant direct interaction in these models (de Reffye et al., 2008). However, the lack of trophic competition simulation may hinder the simulation of feedback effects of biomass partitioning on other processes. As Pallas et al. (2008) state, trophic competition influenced the organogenesis of grapevine in their research". That paragraph had been deleted by you in last revision. Do you think we should still mention somewhere this idea "mechanistic sinksource solver helps the simulation of biomass partitioning, and also the future possibility of simulating feedback effects"?. I added one sentence in the discussion to add about considering feedback effects:" 'Introducing a mechanism of trophic competition at organ level in a PBM, as done in this study, opens the possibility to model feedbacks effects of biomass partitioning on other 
processes such as photosynthesis or organogenesis (Mathieu et al., 2009). ". Do you agree? Moreover, it was shown in Lecoeur et al. (2011) that $H I$ is the parameter that contributes the most (14.3\%) to the coefficient of variation of the potential grain yield. It was also shown that when ranking the processes in terms of their impact on yield variability, the first one was biomass allocation (before light interception according to plant architecture, plant phenology and photosynthesis). Therefore, Lecoeur et al. (2011) suggested that a better formalisation of the trophic competition between organs could be a way to improve our understanding of genotypic variation for the harvest index Fenni: here the third reviewer posed another question last time "Please elaborate and give appropriate references to clarify the idea of formalizing trophic competition (direct formalization? It could also be indirect) and to construct a sound argument (e.g. which outlines to satisfy the need of feedback effects of biomass partitioning)." I actually didn't answer his question about direct formalization or indirectIn fact I don't understand the reviewer's question; what do you think he means by "direct or indirect formalization"'?. In order to take up this challenge, a new sunflower model, named SUNLAB, was derived from SUNFLO. The representation of plant topological development and allocation process at individual organ scale were inspired by the functional-structural plant model GREENLAB, which has been designed as a "source-sink solver" (Christophe et al., 2008) and which is accompanied with the appropriate mathematical tools for its identification (Cournede et al., 2011). SUNLAB thus inherits from GREENLAB the flexible rules of sink competition for biomass partitioning at organ scale Fenni:the deleted phrases "(organ type includes blade, petiole, intern- 
ode and capitulum; a leaf consists of a blade and a petiole; for the modeling of trophic competition, blade and petiole are considered as two organ types)" were actually trying to answer the second reviewer's question in the previous review "you are using the word "blade" to designate the leaves all over the manuscript. This is somewhere confusing. The manuscript would benefit to clarify this". Maybe somewhere a small explanation of blade could be added if it is deleted here Yes, I had taken care of that and I had added it in the MM part, in plantstructure paragraph as "' The different organ types, denoted as o, include leaves (decomposed into blades and petioles)," Do you think that it will be ok ?, together with the more detailed representation of ecophysiological processes and environmental stress effects on biomass production and yield from SUNFLO.

This paper presents in detail the mechanisms of SUNLAB and the parameter estimation procedure based on field experimental data. A sensitivity analysis is performed on the model parameters, using the Sobol method, to investigate the relative contribution of each parameter and their interactions to the model output uncertainty. The output that we consider is the main trait of interest in most breeding procedures, that is the final grain yield. The potentials of SUNLAB for genotypic characterization are illustrated by comparing the parameters obtained after the estimation process for four genotypes, namely "Albena", "Heliasol", "Melody" and "Prodisol". The performances of SUNLAB to reproduce phenotypic variability coming either from genotypic or from environmental influences are tested against experimental datasets used for parameterization. An additional dataset is then used for model evaluation. An interesting and uncommon output of 
SUNLAB is the simulation of specific leaf area (SLA, also known as leaf specific surface area, $\left.\mathrm{cm}^{2} \cdot \mathrm{g}^{-1}\right)$, i.e. the ratio of leaf area to dry leaf mass. It is an influent input variable often associated with large uncertainty ranges in most dynamic crop growth models (Rawson et al., 1987).

\section{Materials and methods}

\subsection{Modeling: SUNLAB modules}

SUNLAB consists of five modules: phenology, water budget, organogenesis and morphogenesis, biomass accumulation, and biomass partitioning. Phenology, water budget, and biomass accumulation modules are directly inherited from the SUNFLO model. The organogenesis and morphogenesis module is modified from the corresponding SUNFLO module by defining for each organ the dates, expressed in thermal time, of initialization, termination of its growth, and organ expansion. The biomass partitioning module is an entirely new module. We describe here equations of these modules, briefly for those inherited from SUNFLO - we refer to Casadebaig et al. (2011) and Lecoeur et al. (2011) for an exhaustive description - and in detail for the new contributions. Model parameters that are mentioned in the following equations will be summarized in section 2.3.

\subsubsection{Phenology}

Plant phenology is driven by thermal time. Cumulative thermal time on day $d$ since emergence, $C T T(d)\left({ }^{\circ} \mathrm{C}\right.$ days $)$, is calculated in equation (1) as the sum of the daily mean air temperature $T_{m}(d)\left({ }^{\circ} \mathrm{C}\right)$ above a base temperature $T_{b}$ of $4.8{ }^{\circ} \mathrm{C}$, common to all sunflower genotypes. Four key phenological stages, expressed as genotype-dependent thermal dates $\left({ }^{\circ} \mathrm{C}\right.$ days $)$, 
are defined: flower bud appearance $E 1$, beginning of flowering $F 1$, beginning of grain filling $M 0$ (early maturation) and physiological maturity $M 3$ (Lecoeur et al., 2011). Crop development can be accelerated by water stress, that causes overheating of the plant through the reduction of transpiration. This is modeled by using a multiplicative effect of water stress at day $d$, FHTR(d)(the effect of water on transpiration), on thermal time accumulation $C T T(d)$ :

$$
\begin{aligned}
& T_{\text {eff }}(d)=\max \left(0,\left(T_{m}(d)-T_{b}\right)\right) \\
& C T T(d)=\sum_{k=1}^{d} T_{\text {eff }}(k) \times[1+0.1 \times(1-F H T R(k))]
\end{aligned}
$$

where $T_{\text {eff }}(k)$ is the effective thermal time at day $k$. FHTR $(d)$ is calculated as a function of the fraction of transpirable soil water at day $d, F T S W(d)$ (detailed in 2.1.2), divided by a genotypic parameter $R T$ of sensitivity to water deficiency (Casadebaig et al. (2011)).

\subsubsection{Water budget}

In SUNLAB, the water cycle of sunflower is modeled considering the plant processes (root water absorption and transpiration), in combination with its direct environment: precipitation, irrigation, soil evaporation (see Fig. 1a). Evaporation and plant transpiration decrease the available amount of water in soil, while irrigation and precipitation refill it.

[Figure 1 about here.]

The index for the assessment of drought level $\operatorname{FTSW}(d)$ at day $d$ depends on the simulation of the above mentionned processes (Lecoeur et al., 2011). It takes values from 0 (no water stress) to 1 (severe water stress) and it is 
used to define three indices to tune three plant functioning processes: leaf expansion FHLE, radiation use efficiency FHRUE and plant transpiration FHTR. The critical thresholds $R T$ and $R O$ are genotype-dependent parameters varying in $[0,1]$ that characterize the plant drought tolerance $(R T$, drought tolerance of leaf expansion; $R O$, drought tolerance of radiation use efficiency and transpiration). For instance, FHRUE is calculated as:

$$
F H R U E(d)= \begin{cases}F T S W(d) / R T & \text { for } \quad \operatorname{FTSW}(d)<R T \\ 1 & \text { for } \quad \operatorname{FTSW}(d) \geq R T\end{cases}
$$

Its effect on radiation use efficiency $R U E(d)\left(g \cdot M J^{-1}\right)$ is defined as:

$$
R U E(d)=R U E_{p}(d) \times \min \left(1, \frac{F T S W(d)}{R T}\right) \times F T(d) \times P H S
$$

where $R U E_{p}(d)\left(g \cdot M J^{-1}\right)$ is the crop potential (maximal) radiation use efficiency, $F T(d)$ is the thermal stress on day $d$, a function of daily mean temperature (Lecoeur et al., 2011) and PHS is a genotypic parameter giving the ratio of the genotype photosynthesis capacity to that of the reference genotype "Melody". The potential radiation use efficiency is thus weakened by the environmental thermal stress factor $F T(d)$ and the drought stress factor $\operatorname{FHRUE}(d)$.

\subsubsection{Organogenesis and morphegenesis}

Ecophysiological functions. The number of blades $N(d)$ on day $d$ increases linearly with cumulative thermal time $C T T(d)$ :

$$
N(d)=R \times C T T(d)+1
$$

where $R$ (leaves/ ( ${ }^{\circ} \mathrm{C}$ days $)$ ) is the rate of leaf production. Leaf senescence occurs during the period of grain filling between M0 and M3. Consequently 
the number of senescent leaves $N S(d)$ is considered to increase in proportion to the time elapsed since M0 (Sinclair and de Wit, 1975; Nooden et al., 1997) as:

$$
N S(d)=N_{\text {total }} \times \frac{M 3-C T T(d)}{M 3-M 0}
$$

where $N_{\text {total }}$ is a genotypic parameter equal to the maximal number of leaves. Since, in sunflower, leaf area distribution along the stem shows a bell shape, total leaf area $A(d)\left(\mathrm{cm}^{2}\right)$ per plant is calculated with a logistic equation:

$$
A(d)=\frac{A 1}{1+e^{4 \times A 3 \times(A 2-N(d)) / A 1}}
$$

where $A 1\left(\mathrm{~cm}^{2}\right)$ is the maximal leaf area, $A 2$ and $A 3\left(\mathrm{~cm}^{2}\right)$ are respectively the rank and the area of the largest leaf of the plant. The calculation of senescent leaf area $A S(d)\left(\mathrm{cm}^{2}\right)$ is determined by a similar logistic equation but replacing $N(d)$ by $N S(d)$. The photosynthetically active leaf area $A A(d)$ $\left(\mathrm{cm}^{2}\right)$ is estimated as the difference between total leaf area $A(d)$ and senescent leaf area $A S(d)$. Leaf growth and senescence are affected by water stress and temperature stress coefficients as described in Casadebaig et al. (2011).

$$
A A(d)=\frac{A 1}{1+e^{4 \times A 3 \times(A 2-N(d)) / A 1}}-\frac{A 1}{1+e^{4 \times A 3 \times(A 2-N S(d)) / A 1}}
$$

Plant structure. The different organ types, denoted as $o$, include leaves (decomposed into blades and petioles), internodes and capitulum. For each individual organ $o$ at rank $i$ ( $i$ takes its values from 0 to the total amount of individual organs of type $o$ ), its emergence thermal time $\operatorname{initTT}(o, i)$, senescence thermal time seneTT $(o, i)$, and growth expansion duration in thermal time $\operatorname{epdTT}(o, i)$ are defined. The thermal time of blade emergence and 
senescence are calculated through inversion of equations 4 and 5:

$$
\begin{aligned}
& \text { initTT }(\text { blade }, i)=(i-1) / R \\
& \text { seneTT }(\text { blade }, i)=M 3-\frac{i \times(M 3-M 1)}{N_{\text {total }}}
\end{aligned}
$$

For the calculation of blade expansion duration epdTT(blade, $i$ ), three parameters initTT Adjust $\left({ }^{\circ} \mathrm{C}\right.$ days $)$, epdTT $A\left({ }^{\circ} \mathrm{C}\right.$ days $)$, epdTTB $\left({ }^{\circ} \mathrm{C}\right.$ days $)$ are added to the module to calculate epdTT(blade, $i$ ) based on the blade emergence and senescence thermal times:

$$
\begin{aligned}
& \operatorname{epdTT}(\text { blade }, i)=\operatorname{seneTT}(\text { blade }, i)-(\operatorname{epdTTB}-\operatorname{epdTTA} \times i) \\
& \text {-(initTT(blade, } i) \text { - initTTAdjust) }
\end{aligned}
$$

Since leaf emergence was recorded when lengths of their central vein are bigger than 4cm (Lecoeur et al., 2011), the leaf has already received a small amount of biomass at the recorded thermal time initTT(blade, $i$ ). Therefore, thermal time of blade growth initialization is calculated by subtracting initTT Adjust to the emergence thermal time initTT(blade, $i$ ). The thermal times of end of blade expansion linearly vary with their ranks and depend on two parameters, epdTT $A$ and epdTTB.

The petiole at rank $i$ shares the same initial, senescence and expansion thermal times as the blade $i$ belonging to the same metamer. The internode $i$ has also the same emergence thermal time as the blade $i$ while its expansion duration epdTT (internode, $i)$ is driven by a parameter internode EpdTT that is common to all internodes. Capitulum initialization thermal time corresponds to $M 0$. Its expansion duration is defined by the parameter capitulumEpdTT. These additional parameters to the module are estimated as described in 2.3 . 
With all the information of emergence and senescence thermal times of every organ, a general sunflower structure can be constructed. Their expansion durations are important variables for the calculation of biomass distribution to organs as presented in 2.1.5.

\subsubsection{Biomass accumulation}

Daily increase in above-ground dry matter $D M(d)\left(g \cdot m^{-2}\right)$ is calculated from Monteith's equation (1977) linking dry matter production to incoming photosynthetically active radiation through two radiation efficiencies as follows:

$$
D M(d)=R U E(d) \times R I E(d) \times P A R_{0}(d)
$$

where $P A R_{0}(d)\left(M J . m^{-2}\right)$ is the daily incident photosynthetically active radiation. $R U E(d)\left(g \cdot M J^{-1}\right)$ is daily radiation use efficiency and $R I E(d)$ is daily radiation interception efficiency, estimated from Beer's law. The total above-ground biomass $C D M(d)\left(g \cdot m^{-2}\right)$ is the cumulated daily biomass production from emergence:

$$
C D M(d)=\sum_{k=1}^{d} D M(k)
$$

\subsubsection{Biomass partioning}

As in GREENLAB, the biomass produced by leaves is distributed to all organs proportionally to their respective demands. Indeed, it has been observed for several crops that the final balance of the source and sink relationships in the end is similar to the action of a common pool of biomass (e.g. Heuvelink (1995) for tomato): this simplification enables skipping the details of the transport resistance system and other complex features of branching 
systems (Christophe et al., 2008). The biomass is dynamically distributed to every "sink" organ, including blades, petioles, internodes and the capitulum, regardless of their position within the plant structure. Blades are "sources" whose photosynthetic production fills the pool biomass. The calculation of the daily incremental mass of each organ is done through three steps.

First step: Definition of individual organ sink. Biomass is partitioned to organs according to their number, age and relative sink strength. The relative sink strength of organs of given type $o$ is denoted as $S R(o)$, which is a dimensionless variable indicating the ability of different kinds of organs in competing for biomass. The relative sink strength of all blades is set to 1 as a reference value, i.e. $S R($ blade $)=1$ (Kang et al., 2008). The growth rate of an individual organ can vary through its expansion period. This change is modeled by a normalized discrete Beta density function in GREENLAB model (Kang et al., 2008) and in this model. Among any empirical functions that could be suitable, the Beta function is recommended by Yin et al. (2003) as it presents several advantages: at intial and final times, its values are zero, it has a high flexibility and can describe asymmetric growth trajectories and it has stable parameters for statistical estimation. Therefore, the actual sink strength of an organ $S A P(d, o, i)$ (e.g. the actual sink strength an organ of type $o=$ blade, at rank $i=2$, on day $d S A P(d$, blade,2)) can be expressed as:

$$
\begin{aligned}
& S A P(d, o, i)=\left(1-\frac{C T T(d)+\operatorname{initTT}(o, i)}{\operatorname{epdTT}(o, i)}\right)^{\operatorname{sinkB}(o)-1} \\
& \times\left(\frac{C T T(d)-\operatorname{initTT}(o, i)}{\operatorname{epdTT}(o, i)}\right)^{\operatorname{sink} A(o)-1} \times \frac{S R(o)}{M(\operatorname{sink} A(o), \operatorname{sink} B(o))}
\end{aligned}
$$


where $M(A, B)$ is a normalization factor defined as:

$$
M(A, B)=\left(\frac{A-1}{A+B-2}\right)^{A-1} \times\left(1-\frac{A-1}{A+B-2}\right)^{B-1}
$$

Two organ-type-specific parameters $\operatorname{sink} A(o)$ and $\operatorname{sink} B(o)$ control the function shape, as illustrated in the result section Fig. 4. Thus, the sink activity of an organ $o$ at rank $i$ starts from $\operatorname{initTT}(o, i)$ and lasts during the organ's expansion duration epdTT $(o, i)$

Second step: Total demand. The plant total demand sumSink $(d)$ is computed as the scalar product of the number of existing organs by their sink strength $S A P(d, o, i)$ :

$$
\operatorname{sumSink}(d)=\sum_{t} \sum_{i} S A P(d, o, i)
$$

Third step: biomass partitioning to organs. The total dry biomass $C D M(d)$ that is produced at day $d$ is allocated to every individual organs proportionnally the ratio of their sink strength $S A P(d, o, i)$ to the total plant demand sumSink $(d)$. For example the biomass allocated to an individual blade indMS $(d$, blade,$i)\left(g . m^{-2}\right)$ of blade ranking $i$ is:

$$
\text { ind } M S(d, \text { blade }, i)=\frac{C D M(d) \times S A P(d, \text { blade }, i)}{\operatorname{sum} \operatorname{Sink}(d)}
$$

Total blade biomass organMS(d,blade) $\left(g \cdot m^{-2}\right)$ at time $d$ is the sum of all individual blade biomass:

$$
\operatorname{organ} M S(d, \text { blade })=\sum_{i} \operatorname{ind} M S(d, \text { blade }, i)
$$

Similarly, individual and total petiole biomass (indMS $(d$, petiole, $i)$ and organ $M S(d$, petiole), $g . m^{-2}$ ) are simulated, as well as individual and total internode biomass (indMS $(d$, internode, $i)$ and organ $M S(d$, internode $\left.), g \cdot m^{-2}\right)$, and capitulum biomass $\left(i n d M S(d\right.$, capitulum, $i)$ organ $M S(d$, capitulum $\left.), g . m^{-2}\right)$. 


\subsection{Field experiments and measurements}

Experiments and measurements for designing and constructing modules which are directly inherited from SUNFLO are not presented in this paper, as they are described in detail in Lecoeur et al. (2011). Data used for SUNLAB parameters estimation, simulation and application include three datasets, respectively entitled as "2001", "2002a" and "2002b". They all come from field experiments conducted in 2001 and 2002 at SupAgro experimental station at Lavalette $\left(43^{\circ} 36^{\prime} \mathrm{N}, 3^{\circ} 53^{\prime} \mathrm{E}\right.$, altitude $\left.50 \mathrm{~m}\right)$ on a sandy loam soil for four genotypes "Albena", "Heliasol", "Melody" and "Prodisol". In "2001", Sunflowers were sown on 5 May 2001 at a density of about 6 plants $m^{-2}$ and a row spacing of $0.6 \mathrm{~m}$, in a randomized complete block design with four replications. Plots measured 5.5. $\times 13.0 \mathrm{~m}$. In the other two datasets, experiments were conducted with the same plant arrangement. But sunflowers were sown on 15 May 2002 and plots measured $8.0 \times 8.0 \mathrm{~m}$. During the experiment, meteorological data such as temperatures and radiation were recorded. The total amount of water available for the plant was calculated as the difference between soil water content at field capacity estimated at the beginning of the experiment and soil water content at $10 \%$ of maximal stomatal conductance. The fraction of transpirable soil water $(F T S W)$ remaining in the soil at a given date was calculated as the ratio of actual plant-available soil water content to the total plant-available soil water content (Lebon et al., 2006). Organogenesis was described based on the phenomenological stages that were recorded every 2-3 days (Lecoeur et al., 2011). Once a week, six plants per genotype were harvested. Individual leaf areas were estimated from blade lengths and widths. All the above-ground organs (blades, peti- 
oles, stem, capitulum and seeds) were collected and then oven-dried at $80^{\circ} \mathrm{C}$ for $48 \mathrm{~h}$. The dry weights of these organs were measured by compartments. Daily radiation interception efficiency $R I E(d)$ and daily radiation use efficiency $R U E(d)$ were respectively calculated and estimated based on field measurements as in (Lecoeur et al., 2011). In all experiments, the crop was regularly irrigated and fertilized to avoid severe water deficits and mineral deficiency. But in practice, the three experiments showed different water deficit conditions. The index FTSW of the three experiments, which can represent the water stress level, is illustrated (Fig.2). Since the experiment measurements were carried out every a few days, an interpolation on experimental data was drawn to make the contrast clearer. Datasets "2001" and "2002a" correspond to contrasted environmental conditions and are used to calibrate SUNLAB model while "2002b" is used for model evaluation.

[Figure 2 about here.]

\subsection{Parameter analysis}

Four genotypes "Albena", "Melody", "Heliasol" and "Prodisol" are considered in this paper. These genotypes have been characterized by a large study of genetic improvement of sunflower over the last 30 years, and they are four of those most widely grown varieties in France from 1960 to 2000 (Vear et al., 2003). SUNLAB parameters can be decomposed in two subsets. One subset contains the parameters inherited from SUNFLO which keep the same values in SUNLAB (Table 1).

[Table 1 about here.] 
The other subset contains 17 additional parameters, that are introduced in SUNLAB, as was presented in section 2.1. They include 12 parameters that drive the sink competition ( $S R, \operatorname{sink} A, \operatorname{sink} B$ for four types of organs) and 5 parameters, that are used to adjust or define initial and final organ expansion thermal times: initTT Adjust $\left({ }^{\circ} \mathrm{C}\right.$ days $)$, epdTTA $\left({ }^{\circ} \mathrm{C}\right.$ days $)$, epdTTB $\left({ }^{\circ} \mathrm{C}\right.$ days), internodeEpdTT $\left({ }^{\circ} \mathrm{C}\right.$ days $)$, and capitulumEpdTT $\left({ }^{\circ} \mathrm{C}\right.$ days $)$. Note that the sink strength of blades $S R$ (blade) is set to 1 as a reference value (Christophe et al., 2008), therefore only 16 parameters are included in the sensitivity analysis and estimation procedure.

The non-linear generalized least squares method with Gauss Newton algorithm for optimization (Cournede et al., 2011) was used for estimating the 16 parameters of four genotypes. The target field data include (i) total blade mass, total petiole mass, total internode mass, and capitulum mass, all collected once a week during 15 weeks in total, and (ii) individual blade mass. Regarding the target field data at organ scale used for parameter estimation, only individual blade area data was available. All organs were only weighted at compartment scale. In particular, independent blade mass data was not available, while these data are required for a better estimation of SUNLAB parameters. Therefore, profiles of individual blade mass were estimated as follows: at each date when total blade mass and total blade areas were measured at compartment level, a virtual $S L A$ value was computed as the ratio of these two quantities and was used to generate a set of individual blade mass from the sequence of areas. The model can thus be viewed as a dynamic interpolation solver that generates both blade areas and mass between those fixed measurement dates. Since these measurements at individual scale were 
performed 6 times, the estimated blade mass represent around 150 data for each genotype, to be added to the 60 data at compartment scales, giving a total of around 210 observation data used for the parameter estimation of each genotype.

A sensitivity analysis was performed on SUNLAB parameters to understand their relative influence on determining the main model output, the yield $Y$. A global method was used, the Sobol method (Saltelli et al., 2000; $\mathrm{Wu}$ and Cournede, 2010). In this method, parameters are considered as random variables that are drawn from predefined distributions, chosen here as uniform distributions since no a priori knowledge is available for the 16 SUNLAB parameters. Plausible interval boundaries are defined: the lower boundary is set as 0.5 times of the parameter's minimum estimated value among all genotypes, and the upper boundary is set as 1.5 times of the parameter's maximum estimated value. This allows computing an estimator of the output variance, $V(Y)$. The first-order sensitivity index of a given parameter $X_{i}$ can thus be defined as:

$$
S_{i}=\frac{V_{X_{i}}\left(E_{\sim X_{i}}\left(Y \mid X_{i}\right)\right.}{V(Y)}
$$

where the inner expectation operator is the mean of $Y$ taken over the possible values of all other parameters except $X_{i}\left(E_{\sim X_{i}}\right)$ while keeping $X_{i}$ fixed. Then outer variance is taken over all possible values of $X_{i}$. Similarly, higher order sensitivity indices can be defined to characterize the effects of interactions between parameters on the output variance. Sensitivity indices are normalized thanks to the well-known formula of variance decomposition. Here, 1000 parameter sets are generated from the Sobol sequence in the calculation. 
This crop model SUNLAB and the statistical analysis methods are integrated in the platform PYGMALION (Cournède et al., 2013): this platform is currently developed and used in the laboratory of Applied Mathematics and Systems at Ecole Centrale Paris, and is available to a few other labs for collaborative research projects. Programmed in $\mathrm{C}++$ computer language, it is dedicated to the mathematical analysis of plant growth models, including the parameter estimation and sensitivity analysis methods used in this paper. It comprises approximately 20 classical and new models of plant growth, among which are Greenlab (Hu et al., 2003), PILOTE (Mailhol et al., 1997, 2004), STICS (Brisson et al., 1998), SUNFLO (Casadebaig et al., 2011) and SUNLAB.

\section{Results}

\subsection{Sensitivity analysis}

A sensitivity analysis was performed on the 16 parameters (described in 2.3) of SUNLAB for the yield, using the Sobol method of variance decomposition. Results are gathered in Table 2 for the most influential parameters. The sum of all first order indices was 0.87 , which means that the part of variance due to parameter interactions was less than 15\%: this justifies that the sensitivity analysis of this model can be grounded on first-order indices of parameters. The most influential parameters are those driving the dynamics of capitulum sink variations, $\operatorname{sink} A(c a p)$ and $\operatorname{sink} B(c a p)$, accounting for $51 \%$ and $12 \%$ respectively of the yield variance. The only other parameter with significant sensibility index is a parameter of internode sink variation, sinkA(intern). All other parameters account for less than $5 \%$ of the yield 
variance. This result suggests that dynamics of biomass allocation to the capitulum, more than the value of its sink, are important for yield determination.

[Table 2 about here.]

\subsection{Model parameterization}

\subsubsection{Parameter estimation for four sunflower genotypes}

The SUNLAB parameters were estimated for the four different genotypes ("Albena", "Melody", "Heliasol", and "Prodisol") using experimental datasets of "2001" (non-limiting conditions) and "2002a" (with water deficit). The values of the 12 sink competition related parameters are shown in Table 3 with the associated standard deviation.

These parameter values were independently estimated for each genotype, i.e. no a priori genotypic correlations were imposed. This allows comparing the genotypes according to their parameter values. The standard error could allow testing the significance of differences between two parameter values, but this would only be an approximate result since the number of observations that directly influence the estimation of each parameter was unknown. to change with the results of the test. Qualitative observations can nevertheless be done. For example, blade parameter sinkA(blade) in the sink variation function of blades appears significantly different between four genotypes, while no clear evidence of genotypic variability was found for capitulum sink strength ratio $S R$ (capitulum) (see also Fig.4). The internode sink ratio, $S R$ (internode), was found different for genotypes "Albena" and "Melody", but took similar values for "Heliasol" and "Prodisol". 
[Table 3 about here.]

\subsubsection{Model performances: reproducing genotype-induced variability}

Even when grown under non-limiting controlled conditions, the four studied varieties presented some phenotypic variability, that might be intrinsically regulated by genotypic influences. This phenotypic variability was in particular observed on daily radiation interception efficiency $R I E(d)$, total blade area $A A(d)$, leaf number $N(d)$, cumulated dry biomass $C D M(d)$ and biomass partitioning. This is illustrated in Fig. 3 for dry mass compartments (blade, internode and capitulum) with the "2001" experimental dataset. This figure also illustrates the model ability to reproduce this (presumably) gentoypic variability.

[Figure 3 about here.]

The estimated parameter values (Table 3) allow tracking back the dynamics of biomass allocation and analyzing the internal mechanisms underlying sink competition. For instance, compared to "Prodisol", blades of "Albena" entered earlier in the competition for biomass but the capitulum reached its maximum demand later (Fig.4): this may explain that in the end "Albena" had bigger total blade biomass but smaller capitulum biomass than "Prodisol" (Fig. 3). Genotypic characterization can also come from the biomass accumulation module: "Melody" had larger internode and capitulum biomass than "Heliasol", and they had similar blade biomass, as can be seen in Fig. 3. This was due to a higher radiation use efficiency of the "Melody" genotype.

[Figure 4 about here.] 


\subsubsection{Model performances: reproducing environment-induced variability}

The SUNLAB model was calibrated using "2001" and "2002a" experimental datasets that included data for plants grown without water deficit ("2001") and plants grown under water deficit ("2002a"). The parameterized SUNLAB model was able to simulate the phenotypic variability induced by the two contrasted environmental conditions of "2001" and "2002a" datasets. This is illustrated in Fig.5 that shows experimental data and simulations of radiation interception efficiency $R I E(d)$, total blade area $A A(d)$, leaf number $N(d)$, cumulated dry above-ground biomass $C D M(d)$ and biomass compartments (capitulum, blades, petioles, internodes) for the "Melody" genotype. It can be noticed that "Melody" was not very sensitive to water stress since the dry mass accumulation did not significantly vary. Graph B shows that there were under-estimations of total blade area. This was due to the modeling equations of leaf area (see equation 6 and equation 7 ). These equations are inherited from SUNFLO model and define a common formula for all genotypes to calculate total leaf area based on genotype-specific parameters $A 2$ and $A 3$. This common formula does not allow to account for all the genotypic variance of total leaf area: possible improvements on this part of the model are discussed in section 4. Graph E and Graph F of this Fig.5 present some details on two other genotypes: biomass compartments of "Prodisol" and individual blade mass profile for "Heliasol". Water stress induced a decrease in the capitulum biomass of "Prodisol" plants, despite a slight increase in blade biomass. The effect of water stress can also be observed on the individual blade mass profile of "Heliasol" plants: blades on the last ranks grew less in water deficit conditions ("2002a") than in standard conditions ("2001"). 
[Figure 5 about here.]

\subsection{Model evaluation}

In order to test the model predictive ability, SUNLAB was confronted to an additional experimental dataset "2002b", that was not used for the parameterization step. Fig. 6 presents some phenotypic traits for the "Albena" genotype: for total blade areas and radiation interception efficiency, data were underestimated by model predictions, total dry biomass was also proportionally affected, but the results were reasonable for the biomass compartment dynamics. The root mean square error (RMSE) of organ mass for genotype "Albena", calculated on days with available experimental data, was 36.4 and its coefficient of determination was 0.95 . However, it has to be noticed that this evaluation process was still at a preliminary step since our additional experimental dataset "2002b" was measured in experimental conditions similar to those of the "2002a" dataset which was used to calibrate the model.

[Figure 6 about here.]

\subsection{Model Application: an exploratory study on specific leaf area}

Specific leaf area (SLA) is an important variable in plant growth modeling. In most dynamic models, it is usually used to determine blade surface area values from blade biomass, as in GREENLAB (Christophe et al., 2008) or in TOMSIM (Heuvelink, 1999). Since blade area in turn determines the biomass production, accurate estimation of SLA is mentioned as a major source of error in models and implies difficulties in obtaining a reliable computation of leaf area index, which is the main component of biomass 
production modules (Heuvelink, 1999; Marcelis et al., 1998). It is however generally considered as constant, although it has been shown, for instance on wheat (Rawson et al., 1987), that SLA varies according to genotypes, leaf ranks and leaf growing periods. Regarding sunflower, the variations of SLA and the factors influencing them are still poorly known. As SUNLAB can simulate dynamics of individual blade mass profiles independently from those of blade areas, the SLA can be computed as a model output, contrary to the classical situation where it is taken as input. In Fig.7, the simulated and observed values for individual blade areas and masses of "Melody" in the "2001" dataset are displayed for each blade rank and six different growth stages.

[Figure 7 about here.]

The SLA was computed at the time when individual blades have reached their highest mass on $67^{\text {th }}$ day. SLAs are illustrated for blades ranking from 9 to 15 which are those whose individual blade mass and area had the best accordance to the field data (Fig. 7). The root mean squared error (RMSE) of SLA for these blades ranking from 9 to 15 was 11 and the coefficient of variation $(\mathrm{CV})$ was $25 \%$. But for all blades on $67^{\text {th }}$ day, including those whose individual blade leaf areas were poorly simulated, the RMSE of SLA for genotype Melody became 35, with CV value $76 \%$. The computed SLA showed some variability among the four genotypes. But since the current SUNLAB parameters came from reconstructed individual blade masses, these simulated SLA results are expected to be improved with better experimental data in the future. Moreover, the modeling of individual leaf area should be improved as well for more accuracy on this result. 


\section{Discussion and conclusion}

Models in the breeding process. After further tests and improvements, this new SUNLAB model should present robust enough predictive capacities and ability to differentiate between genotypes in order to be proposed as a proper tool for the understanding of crop phenotypes induced by genotype $\times$ environment interactions. Practical considerations should also be examined in our context of model application, i.e. transferring model-based information to breeders. This kind of information could be for instance recommendations on optimal environmental conditions or management practices for a given genotype; the identification of particular features (a subset of the model parameters, for instance) to focus on in the breeding process in order to create variants with some targeted traits; environmental characterization for genotypes performances; or the prediction of crop growth and harvest.

SUNLAB has the potential to be used in studying the link between crop model parameters and genetic information. As stated in Messina et al. (2006), the breeding of higher-yielding crop plants would be greatly accelerated if the phenotypic consequences of changes at some genetic markers of an organism could be reliably predicted. Recently, quantitative trait loci (QTL) information has been incorporated into some organ-level crop models (Reymond et al., 2003; Yin et al., 2006; Xu et al., 2011). To address the link between model parameters and QTL, well designed models and suitable experimental data are required. Appropriate model structures allow sufficient physiological feedback features to be incorporated. Model input parameters should be designed to be grounded potentially in gene-level understanding (Yin et al., 2004). It requires the plant growth model parameters 
having biological meaning to represent genetic coefficients (Yin and Struik, 2010; Tardieu, 2003). The organ-level model SUNLAB and its parameters are expected to meet the requirements. In line with what has been done for pepper in Alimi et al. (2013), SUNLAB is considered to be used in a study with an experimental database of 90 sunflower genotypes which are F1 hybrid of the first filial generation resulting from a cross mating of $9 \times$ 10 distinctly different parental types. After estimating SUNLAB parameters for the 90 genotypes, statistical analyses of the correlations between different genotypes' parameters could reveal certain genetic links.

About the modeling approach: from process-based model to functional structural model. The design of the SUNLAB model was based on an ecophysiological model, SUNFLO, that was transformed to a FSPM and enriched with a mechanistic module for biomass allocation to organs. Fenni: in the deleted paragraph "While there exist many excellent PBM models with accurate model identification and growth description, it is possible to convert them into FSPMs to take advantage of FSPMs' structures and organs' interaction, and to reduce the efforts of building a FSPM from blank. Feng et al. (2010) tested using GREENLAB sink-source solver to improve the PBM model PILOTE (Mailhol et al., 1997) for the crop Maize (Zea mays L.) Actually, these previous sentences were already re-used below.. In this paper, SUNLAB is a good demonstration for the crop Sunflower (Helianthus annuus L.). It defines sunflower's structural development and it adds complex biomass partitioning mechanism to SUNFLO, while it keeps certain modules of this PBM model, with the advantages of inheriting its ecophysiological merits...", some sentences were actually trying to answer the first reviewer's 
question in the previous review: "A simple conceptual representation could be useful to better understand how the source-sink model (GREENLAB) integrates with process-based model (SUNFLO)". Do you think we should add some more sentences as the conceptual representation of the integration of FSPM module in SUNLAB?Vero: I am not sure, but I think that what the reviewer meant was that we add a figure with a diagramm of the SUNLAB model, don't you think so? If so, anyway, we have not done it. In process-based models (PBM), plants are usually considered only at the level of organ compartments. Turning them into FSPM allows taking advantage of the simulation of individual organs' growth and of interactions between organogenesis and functioning. FSPMs focuses on the development, growth and function of individual cells, tissues, organs and plants in their spatial and temporal contexts (Godin and Sinoquet, 2005). It is a solution to take into account the plant's architectural development and to extrapolate PBM at organ level by merging the botanical knowledge on plant development with the functional equations (de Reffye et al., 2008). Introducing a mechanism of trophic competition at organ level in a PBM, as done in this study, opens the possibility to model feedbacks effects of biomass partitioning on other processes such as photosynthesis or organogenesis (Mathieu et al., 2009).

A more classical way to construct FSPM consists in integrating functioning processes into an existing architectural model. This was done for instance for trees in the AMAP- suite (Barczi et al., 2008), for grappevine in Pallas et al. (2011) based on the relationships defined for organogenesis in Lebon et al. (2004), or for wheat in Evers et al. (2010) who built a FSPM from the ADEL-wheat model. Once plant architecture is simulated, incorporating 
functional processes arise as a natural subsequent step in model development. In particular, these 3D-mock-ups are often used to compute light interception. In contrast, since SUNLAB originates from a PBM, the emphasis is put on modeling plant functioning, phenology and effects of water and thermal stresses, while light interception is modeled in a rather simplistic way not relying on the exact 3D structure. A similar approach was applied for the development of the Ecomeristem model of rice growth (Luquet et al., 2006) that incorporates some features (carbon supply, simulation of an initial carbon reserve pool and the mobilisable fraction thereof) of a simple crop model SARRA-H (Dingkuhn et al., 2003). Feng et al. (2010) also tested using the GREENLAB sink-source solver to improve the PBM model PILOTE (Mailhol et al., 1997) for the crop Maize (Zea mays L.).

Generally, our approach fits into a current general trend of development of modular models, with generic modules that can be shared by other modelers. This trend goes hand in hand with the increasing number of modeling platforms: Pygmalion in our case (Cournède et al., 2013), OpenAlea (Pradal et al., 2004), GroIMP (Kniemeyer et al., 2006), etc. These platforms provide flexible frameworks for the coupling of models or the re-use of modules in different models. It reduces the efforts of building models from blank and mutualizes the implementation work. SUNLAB falls within that trend since most of its modules are generic and could be easily adapted to other crops (e.g. biomass allocation module, biomass production module, water budget,...).

Mechanistic modeling and empirical modeling. SUNLAB is the fruit of an effort to make the SUNFLO model more mechanistic (through the modeling of 
biomass partitioning). Mechanistic models generally arise from approaches relating to the complex system theory: they consider the individual components of the system and their interactions, and what emergent properties appear. They have the potential to be used out of their calibration interval, provided that the model predictive capacities have been preliminarily checked. In contrast, empirical models are derived on direct descriptions of observed data. They are usually regression based and provide a quantitative summary of the observed relationships among a set of measured variables. Most plant growth models combine in fact both modeling approaches as a mixture of mechanistic modules and empirical modules.

It is expected that mechanistic description of ecophysiological processes improves the model predictive capacities and their ability to differentiate between genotypes (Allen et al., 2005; Minchin and Lacointe, 2005; Bertheloot et al., 2011). However, the extent to which more mechanistic models are necessarily better should be questioned. In particular, since the parameters in mechanistic modules are assumed to have assigned biological meanings and to represent properties of real system components, the reliability of the underlying assumptions need to be carefully validated. I kept this sentence, but I am not sure of what you meant, Fenni. Could you explain me? Fenni: because the mechanistic models normally try to simulate the biological hypothesis, the parameters in mechanistic modules have assigned biological meanings. Therefore, it need more scrutiny to determine whether the hypothesis and the biological meanings are true. I got this sentence from this article: Biomedical Applications of Computer Modeling, Chapter 7.2 Empirical or mechanisticvro: ok. Do you agree with the way I modified the 
previous sentence, then?. Thus, the appropriateness of mechanistic models needs close scrutiny (Christopoulos and Michael, 2000). Moreover, the parameterization effort of these more and more complex models should always be taken into account when improving their mechanistic description, to prevent from a high level of uncertainty in the parameters which may hinder the original purposes of the model in terms of prediction and genotypic differentiation. So, as stated in the introduction, a delicate trade-off has to be found between mechanistic aspects and complexity, in order to provide proper tools that might be used in the breeding context.

Parameter estimation issue: direct measurements and model inversion. Two kinds of methods were involved for SUNLAB parameterization: estimation through direct measurements and estimation through statistical methods, sometimes referenced as model inversion methods. Direct measurement method enables direct access to the desired parameter via experimental measurements (Jeuffroy et al., 2006). The model inversion method, involving mathematical and statistical calculations, estimates one or more parameters by confronting observed data to simulation results (Guo et al., 2006; Cournede et al., 2011).

Direct measurement is used to estimate parameters that have biological meanings, and that can be directly observable or easily calculated from measured indicators. Parameters with biological meanings consist of two types: "genotypic parameters" which differ between varieties and "crop parameters" which are parameters with small variance among all genotypes. Theoretically, direct measurement method is the best for estimating genotypic parameters and consequently for genotype characterization. The breeder could measure 
it directly on lines under development in experiments in order to predict the expected effects (Reymond, 2001). Similarly crop parameters can be measured directly from field data. Because of the direct and accurate measures on elementary processes, these estimated parameters have advantages in terms of ecophysiological relevance, parameter accuracy and genotype characterization, compared with model inversion method. This perspective has led to automated and high-throughput advanced plant phenotyping (see for example Granier et al. (2005), Sotirios and Christos (2009)). However, the accurate elementary processes do not necessarily imply that the combination of these processes will provide the same accuracy at plant scale. The nonlinear interactions between processes as well as the necessary simplifications in terms of the number of ecophysiological processes considered in the model make the whole plant model not a simple combination of the elementary models that were well calibrated by experiments: plants are complex systems whose description of elementary process interactions, plasticity and robustness remains an open issue (Yin and Struik, 2010). Therefore, parameterization methods relying on model inversion to estimate parameters from experimental data at organ or whole plant levels offers an alternative. This method can ensure an optimized fitting error on training data, but the prediction error on validation data has to be carefully checked to avoid over-fitting problems. The parameters thus obtained have the risk to be less relevant for their biological meanings than direct measurement, because these parameters values may be altered by the error compensation from fitting whole plant processes and from other simultaneously estimated parameters (Jeuffroy et al., 2006). They nevertheless characterize the plant global behavior and may still be 
used to differentiate between genotypes (Letort, 2008).

When parameter estimation is demanded for a high number of genotypes, direct measurement method becomes impractical, because this method often requires specific trials and measurements, which are complicated, costly and even impossible to implement sometimes (Reymond, 2001). Routine measurement of these parameters for a large number of varieties may also pose a problem, particularly when measurements require special equipment and controlled condition experiments (Jeuffroy et al., 2006). Model inversion method is adopted for these cases because it is experimentally less costly and less time-consuming. For instance in most dynamic models, the direct measurement method would often require frequent measurement points (e.g. daily), while with the indirect method, data can be collected only at some given time points and still allow the modellers to retrieve the past growth of the crop. Parameters can even be estimated from very limited sets of data (Kang et al., 2011).

Moreover, some parameters are "hidden",i.e. cannot be experimentally measured and can only be estimated by model inversion method. They usually appear in mechanistic modules, because their underlying mechanisms can produce emergent properties that can be difficult to disentangle $a$ posteriori from the resulting phenotype. It also implies that, because of their interactions, these kinds of parameters cannot be obtained independently from each other: the whole estimation process needs to be performed on all the data at the same time (it is not possible to optimize sequentially on data for different types of organs, for instance).

In SUNLAB, the parameters inherited from SUNFLO have biological 
meaning and had been measured for 20 genotypes. Meanwhile, the parameters involved in the new biomass allocation module are hidden parameters that can only be estimated by model inversion, because the biomass allocation process at organ level is difficult to observe and to be directly measured.

\section{Limitations and perspectives}

Modeling. From the model performances results, we can see that the modeling of blade area needs to be uppermost improved in SUNLAB. A first improvement could consist in replacing the use of the logistic function by a fully mechanistic approach including modeling the SLA instead of deriving it a posteriori from the simulated mass and areas. Thereby, feedbacks effects of trophic competition on leaf area expansion could be explored and modeled.

The biomass accumulation module was directly inherited from SUNFLO that has been tested in different environmental conditions for 26 genotypes (Casadebaig et al., 2011; Lecoeur et al., 2011) and is in line with what is classically done in models of the same class as SUNLAB (e.g. Tomsim (Heuvelink, 1999), Ecomeristem (Luquet et al., 2006)). A more detailed approach, at individual leaf level, could be considered by computing the amount of intercepted radiations: several methods are available (e.g. Nested Radiosity light model in Evers et al. (2010) or a Monte-Carlo radiation model in Xu et al. (2011)) but they require an accurate modeling of the plant structure which is currently not available and would necessitate additional experimental work to be parameterized. It has to be noted that SUNLAB is not stricto-sensu a FSPM since no 3D shape is simulated.

As regards the biomass distribution module that was introduced in our study, our approach is based on the concept of common pool of assimilates 
and relative sink competition. However, some other models (e.g. ECOPHYS [Lacointe et al., 2002])) and experimental observations (Pallas et al. (2008)) suggest that the distance from source to sink could have an influence. An alternative approach is thus to consider transport-resistance methods, as done for instance in the L-PEACH model [Allen et al., 2005]: although these methods are biologically more relevant, they are generally complex and the resulting biomass distribution remains highly dependent on the determination of sink activity. Bancal and Soltani [2002] compared the partitioning coeffcients obtained from an improved version of the transport-resistance model of [Minchin et al., 1993] to the classical sink-based partitioning model: they concluded that the resistance to flux propagation has an influence only in pathologic cases of very low source activity and that resistance terms could be abandoned in most cases as they are only a mathematical burden whose parameter values are very diffcult to measure experimentally. In our sourcesink approach, the main limiting factor is not the geometrical distance but the topological organization of source and sinks (i.e. the number of other sinks in a source-sink pathway) (Letort, 2008).

what do you mean exactly, with this solution?Fenni: I mean the feedback effects of trophic competition on other plant functions could be simulated in the future. It was written in the paragraph you deleted as such "However, the lack of trophic competition simulation may hinder the simulation of feedback effects of biomass partitioning on other processes. As Pallas et al. (2008) state, trophic competition influenced the organogenesis of grapevine in their research. They suggest that a modeling approach simulating sink strength variation and the local effects of sink proximity would be more rele- 
vant than a model considering only development as a function of thermal time or the global distribution of available biomass". I tried to write the modeling limitations in term of blade area modeling, and the modeling of feedback effects of trophic competition. Besides, the third reviewer asked in last review "why the biomass accumulation is a very global level compared to the organ level elsewhere. How do you justify these differences?" I answered him that "The biomass accumulation module is directly inherited from SUNFLO while the biomass distribution module is completely changed, as the first step of adapting it into a FSPM model. Its performance and evaluation have shown satisfactory results. Next step will be to add feedback effects of biomass partitioning to the model, which will improve the simulation of morphogenesis, biomass production etc. This point is discussed in the Discussion and conclusion session in this new version of paper". Therefore I discussed here why feedback effects need to be simulated. This is also my answer to the first reviewer's question "where is the biomass production's under-estimation from" and "why the upper leaves' SLA can not be well simulated". I put the reasons to the bad simulation of blade area, especially the upper leaves' blade area. I mentioned some improvements of biomass production modeling can be planned in the future, such as the feedback effects of biomass distribution on blade area modeling, and also the consequent simulation of biomass production. I also mentioned that to improve the simulation of SLA, "Besides the approach that the logistic function, which is used to model leaf area in SUNLAB, can be compared with other functions, the feedback effect of trophic competition on leaf area expansion can be investigated and modeled." So to sum up, with the limitation of blade area modeling, I tried to answer 
reviewers' three questions: 1, why the biomass production is a very global level compared with biomass distribution; 2, where is the under-estimation of biomass production from; 3 , why did we state that the SLA simulation of upper leaves are not well simulated.

Fenni: the deleted sentences of leaf senescence "In SUNLAB, leaf senescence is modeled to occur between phenology stages $M 0$ and $M 3$. The phenology timing "CTT(d)" is affected by water stress, which affects consequently the rate of leaf senescence. Its leaf senescence start time can be better modeled, since sunflower leaves senescence may occur before $M 0$ stage in drought stressed conditions", is actually an answer to the second reviwer's question in previous review: "Leaf senescence is in the model expected to occur between the stages M0 and M3. In the SUNLAB model, the impact of the drought stress on the phenology is tsaken into account (page 6-line 54 to page 7-line 20); however this point should benefit to be discussed, as sunflower leaves senescence may occur before the M0 stage in drought stressed conditions". I tried to mention the limit of leaf senescence modeling. Sunlab doesn't simulate leaf senescence in strong stress, occuring before M0. Ok, I put some back, then. Do you agree? Besides, leaf senescence is currently affected by water stress only (through the phenology timing "CTT(d)" that affects consequently the rate of leaf senescence) and occurs between phenology stages $M 0$ and $M 3$ while, in reality, it may occur before $M 0$ stage in severe drought conditions. Therefore, the SUNLAB leaf senescence may need also modifications and could include the effects of other environmental cues such as day length and temperature, and various biotic and abiotic sources of stress, that can affect the initiation and progress of leaf senescence (Aguera 
et al., 2012).

Sensitivity analysis. The sensitivity analysis of SUNLAB model in this article has provided parameters' accountability to the variance of crop yield and revealed less than $15 \%$ of effects of parameter interactions in biomass distribution module. It suggests that for the simulation of yield, the empirical modeling could be reasonable, i.e. calculating the yield without the consideration of interactions between the capitulum and other organs' growth. However, the dominant influence of capitulum's sink strength dynamics parameters $\operatorname{sink} A(c a p)$ and $\operatorname{sink} B(c a p)$ may indicate that a better harvest function should be tested rather than a linear relationship with total dry biomass. Sensitivity analysis could also be perfomed on other output of interest such as blade area or stem biomass, in order to better understand the respective influence of the input parameters on the different components of plant phenotype. A sensitivity anlaysis considering all SUNLAB parameters, rather than only parameters in biomass distribution, is also necessary to discover the potential interactions existing among all parameters and modules. A limitation of the Sobol method that was used for these senstivity analyses, is that no correlations were included between the parameters, although they might certainly exist. For example, the correlation analysis of these parameters based on our estimated values in a family of four genotypes indicates a correlation factor of 0.8 for parameters $S R$ (petiole) and $S R$ (internode). For the further characterization of genotypes, sensitivity techniques designed for taking into account input parameters correlations should be adopted $(\mathrm{Xu}$ and Gertner, 2007; Chastaing et al., 2012; Wu et al., 2013). 
Model parameterization and evaluation. Once calibrated, SUNLAB was able of reproducing phenotypic variabilities in different genotypic and environment experiments scenarios. The genotypes "Melody" and "Heliasol" (Fig. 3) were shown to have better drought tolerance than the two other genotypes. Their yields were hardly not influenced by water stress while the other two experienced a slight reduction(around 15\% of 2001 harvest). With some variation according to plant species, certain stages such as germination, seedling or flowering are known to be the most critical stages, vulnerable to water stress (Hadi et al., 2012). Seed germination is the first critical stage and the most sensitive in the life cycle of plants (Ahmad et al., 2009) and seeds exposed to unfavorable environmental conditions, such as water stress at this stage may have seedling establishment compromised (Albuquerque and Carvalho, 2003). However our simulation and field data suggested that drought stress had little effect on crop growth. As sunflower is categorized as a low to medium drought sensitive crop (Turhan and Baser, 2004), the water deficit level might not be strong enough to cause severe growth deficits. An environmental scenario with stronger water deficiency would be required to better parameterize the model. Then, additional scenarios (more cultivar/lines or different kinds of stress conditions) could either help further quantifying the model predictive ability and the range of its validity conditions, or help identifying the inappropriate or missing modules that need further investigation. More importantely, since the model parameters are numerically estimated, these additional scenarios will also allow testing their stability under different environmental conditions and using phenotyping data at different growth stages (Ma et al., 2007, 2008). A good stability is a necessary condition to 
consider these parameters as genotype-dependent and to move forward investigating their potential genetic determinism, as illustrated for example in (Buck-Sorlin et al., 2005).

Fenni: Ok, I agree. This paragraph was added because of the first reviewer's question "Results from model evaluation are not really discussed. I mean, all model are wrong but it is needed to discuss how suitable they are for their use. e.g. Does improving the phenotyping (more cultivar/lines, stress scenarios) automatically increase the prediction capacity when parameter are numerically estimated? Any insights on the actual model performance for discriminating cultivars Vs working with lines?". I didn't answer him about "discrimating cultivars" vs "working with lines", because I don't know the answer. I think in our model, we don't have parameters which can discriminate lines. We only have genotypic parameters, which are cultivardependant, and common parameters for all genotypes. He didn't pose any further question about this point in the new letter, but maybe you could add something to answer his previous question. Ok, see above: I have added the sentences from "'Then, additional scenarios..."': do you agree?"'

Conclusion: Summary of results. A functional-structural model SUNLAB was developed. It describes the sunflower topology and morphogenesis at organ level with blades, petioles, internodes, and capitulum. Coordination of the expansion dynamics of these organs is ruled by their initiation and senescence thermal times. Ecophysiological processes interact with plant structural dynamics to affect biomass accumulation and partitioning to organs. As a joint concept of GREENLAB and SUNFLO models, SUNLAB has better structural features than SUNFLO and it succeeds to deal with the 
biomass distribution at organ level. SUNLAB inherits the ecophysiological merits of SUNFLO that have been validated in different environmental conditions for 26 genotypes (Casadebaig et al., 2011; Lecoeur et al., 2011). In contrast, GREENLAB over-simplifies a number of processes, such as photosynthesis and assimilate conversion to biomass (Guo et al., 2006; Ma et al., 2008), and it is still in its preliminary stage to include water source influence and root system (Li et al., 2009). The ability of this newly-developed SUNLAB model to reproduce observed data of sunflower growth was evaluated on four genotypes "Albena", "Melody", "Heliasol" and "Prodisol".

Aguera, E., Cabello, P., Mata, L., Molina, E., Haba, P., 2012. Metabolic regulation of leaf senescence in sunflower (helianthus annuus l.) plants, senescence. Agricultural and Biological Sciences.

Ahmad, S., Ahmad, R., Ashraf, M., Ashraf, M., Waraich, E., 2009. Sunflower (helianthus annuus l.) response to drought stress at germination and seedling growth stages. Pak J Bot 41 (2), 647-654.

Albuquerque, F., Carvalho, N., 2003. Effect of type of environmental stress on the emergence of sunflower (helianthus annuus l.), soyabean (glycine max (l.) merril) and maize (zea mays 1.) seeds with different levels of vigor. Seed Sci Technol 31, 465-467.

Alimi, N. A., Bink, M. C. A. M., Dieleman, J. A., Nicolai, M., Wubs, M., Heuvelink, E., Magan, J., Voorrips, V., Jansen, J., Rodrigues, P., Heijden, G., Vercauteren, A., Vuylsteke, M., Song, Y., Glasbey, C., Barocsi, A., Lefebvre, V., Palloix, A., van Eeuwijk, F. A., 2013. Genetic and QTL 
analyses of yield and a set of physiological traits in pepper. Euphytica 190, $181-201$.

Allen, M., Prusinkiewicz, P., Dejong, T., 2005. Using L-systems for modeling source-sink interactions, architecture and physiology of growing trees, the L-peach model. New Phytologist 166, 869-880.

Allinne, C., Maury, P., Srrafi, A., Grieu, P., 2009. Genetic control of physiological traits associated to low temperature growth in sunflower under early sowing conditions. Plant Science 177, 349-359.

Barczi, J.-F., Rey, H., Caraglio, Y., de Reffye, P., Barthlmy, D., Dong, Q. X., Fourcaud, T., 2008. Amapsim: A structural whole-plant simulator based on botanical knowledge and designed to host external functional models. Annals of Botany 101 (8), 1125-1138.

Bertheloot, J., Cournède, P.-H., Andrieu, B., 2011. Nema, a functionalstructural model of n economy within wheat culms after flowering: I. model description. Annals of Botany In press.

Brisson, N.and Mary, B., Ripoche, D., M.-H., J., Ruget, F., Nicoullaud, B., Gate, P., Devienne-Barret. F.and Antonioletti, R.and Durr, C., Richard, G., Beaudoin, N., Recous, S., Tayot, X., Plenet, D.and Cellier, R., Machet, J.-M., Meynard, J.-M., Delecolle, R., 1998. Stics : a generic model for the simulation of crops and their water and nitrogen balances. i. theory, and parameterization applied to wheat and corn. Agronomie 18, 311-346.

Buck-Sorlin, G. H., Kniemeyer, O., Kurth, W., 2005. Barley morphology, 
genetics and hormonal regulation of internode elongation modelled by a relational growth grammar. New Phytologist 166 (3), 859-867.

Casadebaig, P., Guilioni, L., Lecoeur, J., Christophe, A., Champolivier, L., Debaeke, P., 2011. Sunflo, a model to simulate genotype-specific performance of the sunflower crop in contrasting environment. Agricultral and forest meteorology 151, 163-178.

Chapman, S., Cooper, M., Podlich, D., Hammer, G., 2003. Evaluating plant breeding strategies by simulating gene action and dryland environment effects. Agronomy Journal 95, 99-113.

Chastaing, G., Gamboa, F., Prieur, C., 2012. Generalized hoeffding-sobol decomposition for dependent variables-application to sensitivity analysis. Electronic Journal of Statistics 6, 2420-2448.

Christophe, A., Letort, V., Hummel, I., Cournede, P., de Reffye, P., Lecoeur, J., 2008. A model-based analysis of the dynamics of carbon balance at the whole-plant level in arabidopsis thaliana. Functional plant biology 35, $1147-1162$.

Christopoulos, A., Michael, J. L., 2000. Beyond eyeballing: Fitting models to experimental data. Critical Reviews in Biochemistry and Molecular Biology 35(5), 359-391.

Cournede, P., Letort, V., Mathieu, A., Kang, M., Lemaire, S., Trevezas, S., Houllier, F., de Reffye, P., 2011. Some parameter estimation issues in functional-structural plant modelling. Mathematical Modeling of Natural Phenomena 6(2), 133-159. 
Cournède, P.-H., Chen, Y., Wu, Q., Baey, C., Bayol, B., 2013. Development and evaluation of plant growth models: Methodology and implementation in the PYGMALION platform. Mathematical Modelling of Natural Phenomena 8, 112-130.

de Reffye, P., Heuvelink, E., Barthlmy, D., Cournde, P.-H., 2008. Plant growth models. In: Jorgensen, S., Fath, B. (Eds.), Ecological Models. Vol. 4 of Encyclopedia of Ecology (5 volumes). Elsevier, Oxford, pp. 2824-2837.

Dingkuhn, M., Baron, C., Bonnal, V., Maraux, F., Sarr, B., Sultan, B., Clopes, A., Forest, F., 2003. Decision support tools for rainfed crops in the Sahel at the plot and regional scales, international fertilizer development center and acp-eu technical centre for agricultural and rural cooperation: wageningen, the netherlands Edition. Struiff Bontekes, T.E. and Wopereis, M.C.S., p. 127139.

Evers, J., Vos, J., Yin, X., Romero, P., van der Putten, P., Struik, P., 2010. Simulation of wheat growth and development based on organ-level photosynthesis and assimilate allocation. Journal of Experimental Botany 61 (8), 2203-2216.

Feng, L., Mailhol, J.-C., Rey, H., Griffon, S., Auclair, D., Reffye, P. D., 2010. Combining a process based model with a functional structural plant model for production partitioning and visualization. 6th International workshop on functional-structural plant models, 41-43.

Godin, C., Sinoquet, H., 2005. Functional-strucutural plant modelling. New Phytologist 166, 705-708. 
Granier, C., Aguirrezabal, L., Chenu, K., Cookson, S. J., Dauzat, M., Hamard, P., Thioux, J.-J., Rolland, G., Bouchier-Combaud, S., Lebaudy, A., et al., 2005. Phenopsis, an automated platform for reproducible phenotyping of plant responses to soil water deficit in arabidopsis thaliana permitted the identification of an accession with low sensitivity to soil water deficit. New Phytologist 169 (3), 623-635.

Guo, Y., Ma, Y., Zhan, Z., Li, B., Dingkuhn, M., Luquet, D., de Reffye, P., 2006. Parameter optimization and field validation of the functionalstructural model greenlab for maize. Annals of Botany 97, 217-230.

Hadi, H., Khazaei, F., Babaei, N., Daneshian, J.and Hamidi, A., 2012. Evaluation of water deficit on seed size and seedling growth of sunflower cultivars. International Journal of AgriScience 2 (03), 280-290.

Hammer, G., Cooper, M., Tardieu, F., Welch, S., Walsh, B., F, F. V. E., 2006. Models for navigating biological complexity in breeding improved crop plants. Trends in Plant Science 11, 587-593.

Heuvelink, E., August 1995. Dry matter partitioning in a tomato plant: one common assimilate pool? Journal of Experimental Botany 46 (289), 10251033.

Heuvelink, E., 1999. Evaluation of a dynamic simulation model for tomato crop growth and development. Annals of Botany 83, 413-422.

Hu, B., de Reffye, P., Zhao, X., Yan, H., Kang, M., 2003. Greenlab: A new methodology towards plant functional-structural model - structural 
aspect. In: Hu, B., Jaeger, M. (Eds.), Plant Growth Models and Applications. Tsinghua University Press and Springer.

Jeuffroy, M.-H., Barbottin, A., Jones, J., Lecoeur, J., 2006. Chapter 10: Crop models with genotype parameters. Working with Dynamic Crop Models, 281-307.

Kang, F., Galinier, T., henry Cournede, P., Lecoeur, J., 2011. Parameterization of plant growth models to characterize genotype by environment interactions: a methodology adapted to breeding programmes. Aspects of Applied Biology, Systems Approaches to Crop Improvement 107, 161-170.

Kang, M.-Z., Evers, J., Vos, J., De Reffye, P., 2008. The derivation of sink functions of wheat organs using the greenlab model. Annals of Botany 101(9).

Kniemeyer, O., Buck-Sorlin, G., Kurth, W., 2006. Groimp as a platform for functional-structural modelling of plants. In: Vos, J., Marcelis, L. F. M., deVisser, P. H. B., Struik, P. C., Evers, J. B. (Eds.), Functional-Structural Plant Modelling in Crop Production. 5.-8. 3. 2006. Springer, Berlin, p. 4352.

Lebon, E., Pellegrino, A., Louarn, G., Lecoeur, J., 2006. Branch development controls leaf area dynamics in grapevine (Vitis vinifera) growing in drying soil. Annals of Botany 98 (1), 175.

Lebon, E., Pellegrino, A., Tardieu, F., Lecoeur, J., 2004. Shoot development in grapevine (vitis vinifera) is affected by the modular branching pattern of 
the stem and intra and intershoot trophic competition. Annals of Botany 93 (3), 263-274.

Lecoeur, J., Poire-Lassus, R., Christophe, A., Pallas, B., Casadebaig, P., Debaeke, P., Vear, F., Guiloni, L., 2011. Quantifying physiological determinants of genetic variation for yield potential in sunflower. sunflo: a model-based analysis. Functional plant biology 38(3), 246-259.

Letort, V., 2008. Multi-scale analysis of source-sink relationships in plant growth models for parameter identification. case of the greenlab model. Ph.D. thesis, Ecole Centrale Paris.

Li, Z., Le Chevalier, V., Cournède, P.-H., November 9-12 2009. Towards a continuous approach of functional-structural plant growth. In: Li, B.-G., Jaeger, M., Guo, Y. (Eds.), 3rd international symposium on Plant Growth and Applications(PMA09), Beijing, China. IEEE.

Luquet, D., Dingkuhn, M., Kim, H., Tambour, L., Clement-Vidal, A., 2006. Ecomeristem, a model of morphogenesis and competition among sinks in rice. 1. concept, validation and sensitivity analysis. Functional Plant Biology 33, 309-323.

Ma, Y., Wen, M., Guo, Y., Li, B., Cournde, P.-H., de Reffye, P., 2008. Parameter optimization and field validation of the functional-structural model greenlab for maize at different population densities. Annals of Botany $101(8)$.

Ma, Y., Zhan, Z., Guo, Y., Luquet, D., de Reffye, P., Dingkuhn, M., 2007. Parameter stability of the structural-functional plant model greenlab as 
affected by variation within populations, among seasons and among growth stages. Annals of Botany 99, 61-73.

Mailhol, J., Olufayo, A. A., Ruelle, P., 1997. Sorghum and sunflower evapotranspiration and yield from simulated leaf area index. Agriculture Water Management 35, 167-182.

Mailhol, J., Zairi, A., Slatni, A., Ben Nouma, B., El Amami, H., 2004. Analysis of irrigation systems and irrigation strategies for durum wheat in tunisia. Agriculture Water Management 70, 19-37.

Marcelis, L., Heuvelink, E., Goudriaan, J., 1998. Modelling of biomass production and yield of horticultural crops: a review. Scientia Horticulturae $74,83-111$.

Mathieu, A., Cournde, P.-H., Letort, V., Barthlmy, D., de Reffye, P., 2009. A dynamic model of plant growth with interactions between development and functional mechanisms to study plant structural plasticity related to trophic competition. Annals of Botany 103 (8), 1173-1186.

Messina, C., Boote, K., Loffler, C., Jones, J., Vallejos, C., 2006. Chapter 11: Model-assisted genetic improvement of crops. Working with Dynamic Crop Models, 309-335.

Minchin, P., Lacointe, A., 2005. New understanding on phloem physiology and possible consequences for modelling long-distance carbon transport. New Phytologist 166, 771-779.

Nooden, L. D., Guiamet, J. J., John, I., 1997. Senescence mechanisms. Physiologia Plantarum 101 (4), 746-753. 
Pallas, B., Loi, C., Christophe, A., Cournde, P. H., Lecoeur, J., 2011. Comparison of three approaches to model grapevine organogenesis in conditions of fluctuating temperature, solar radiation and soil water content. Annals of Botany 107 (5), 729-745.

Pallas, B., Louarn, G., Christophe, A., Lebon, E., Lecoeur, J., 2008. Influence of intrashoot trophic competition on shoot development in two grapevine cultivars (Vitis vinifera). Physiologia Plantarum In press.

Pradal, C., Dones, N., Godin, C., Barbier de Reuille, P., Boudon, F., Adam, B., Sinoquet, H., 2004. Alea: A software for integrating analysis and simulation tools for 3d architecture and ecophysiology. In: 4th International Workshop on Functional-Structural Plant Models. Montpellier, France, p. 406.

Rawson, H., Gardner, P., Long, M., 1987. Sources of variation in specific leaf area in wheat grown at high temperature. Australian Journal of Plant Physiology 14(3), 287-298.

Reymond, M., 2001. Variabilite genetique des reponses de la croissance foliaire du mais a la temperature et au deficit hydrique. combinaison d'un modele ecophysiologique et d'une analyse QTL. These de l'Ecole Nationale Superieure Agronomique de Montpellier, Montpellier, France, 70.

Reymond, M., Muller, B., Leonardi, A., Charcosset, A., Tardieu, F., 2003. Combining quantitative trait loci analysis and an ecophysiological model to analyze the genetic variability of the responses of maize leaf growth to temperature and water deficit. Plant Physiology 131, 664-675. 
Saltelli, A., Tarantola, S., Campolongo, F., 2000. Sensitivity analysis as an ingredient of modeling. Statistical Science 15 (4), 377-395.

Sinclair, T., de Wit, C., 1975. Photosynthate and nitrogen requirements for seed production by various crops. Science 189, 565-567.

Sotirios, T., Christos, N., 2009. Plant phenotyping with low cost digital cameras and image analytics. Information Technologies in Environmental Engineering Environmental Science and Engineering, 238-251.

Tardieu, F., 2003. Virtual plants: modelling as a tool for the genomics of tolerance to water deficit. Trends in Plant Science 8 (1), 9-14.

Turhan, H., Baser, I., 2004. In vitro and in vivo water stress in sunflower (helianthus annuus 1.). Helia 27 (40), 227-236.

Vear, F., Bony, H., Joubert, G., Tourvieille de Labrouhe, D., Pauchet, I., Pinochet, X., 2003. The results of 30 years of sunflower breeding for france. Oleagineux, corps gras 10, 66-73.

Wu, Q., Bayol, B., Kang, F., Lecoeur, J., Cournede, P.-H., 2013. Sensitivity analysis for plant models with correlated parameters: Application to the characterization of sun flower genotypes. 7th International Conference on Sensitivity Analysis of Model Output.

Wu, Q., Cournede, P.-H., 2010. The use of sensitivity analysis for the design of functional structural plant models. Sixth International Conference on Sensitivity Analysis of Model Output 2 (6), 7768-7769. 
Xu, C., Gertner, G., 2007. Extending a global sensitivity analysis technique to models with correlated parameters. Computational Statistics and Data Analysis 51, 5579-5590.

Xu, L., Henke, M., Zhu, J., Kurth, W., Buck-Sorlin, G., 2011. A functionalstructural model of rice linking quantitative genetic information with morphological development and physiological processes. Annals of Botany.

Yin, X., Goudriaan, J., Lantinga, E., Vos, J., Spiertz, H., 2003. A flexible sigmoid function of determinate growth. Annals of Botany 91, 361-371.

Yin, X., Struik, P., Kropff, M., 2004. Role of crop physiology in predicting gene-to-phenotype relationships. Trends in Plant Science 9, 426-432.

Yin, X., Struik, P. C., 2010. Modelling the crop: from system dynamics to systems biology. Journal of Experimental Botany 61 (8), 2171-2183.

Yin, X., Struik, P. C., van Eeuwijk, F. A., Stam, P., Tang, J., 2006. QTL analysis and QTLl-based prediction of flowering phenology in recombinant inbred lines of barley. Journal of Experimental Botany, 1-10. 
${ }_{1150}$ List of Tables

$1151 \quad 1 \quad$ Values of the main parameters inherited from SUNFLO. . . . 52

11522 Sensitivity analysis of SUNLAB parameters: first-order in-

1153 dices of the most influential parameters (with index $>1 \%$ ). . 53

11543 Estimated parameter values of SUNLAB for four genotypes. . 54 
Table 1: Values of the main parameters inherited from SUNFLO.

\begin{tabular}{ccccc}
\hline Parameter & \multicolumn{4}{c}{ Parameter values } \\
\cline { 2 - 5 } Name & Albena & Melody & Heliasol & Prodisol \\
\hline E1 $\left({ }^{\circ} \mathrm{Cd}\right)$ & 510 & 540 & 480 & 510 \\
F1 $\left({ }^{\circ} \mathrm{Cd}\right)$ & 900 & 920 & 880 & 900 \\
M0 $\left({ }^{\circ} \mathrm{Cd}\right)$ & 1160 & 1160 & 1150 & 1120 \\
M3 $\left({ }^{\circ} \mathrm{Cd}\right)$ & 1800 & 2060 & 1940 & 1840 \\
Nmax $(\#)$ & 31 & 26 & 24 & 25 \\
A1 $\left(\mathrm{cm}^{2}\right)$ & 9999 & 9380 & 8707 & 8233 \\
A2 $(\#)$ & 18.9 & 15.4 & 15.3 & 15.9 \\
A3 $\left(\mathrm{cm}^{2}\right)$ & 488 & 613 & 670 & 498 \\
$\mathrm{k}(\#)$ & 0.78 & 0.96 & 0.88 & 0.87 \\
\hline
\end{tabular}


Table 2: Sensitivity analysis of SUNLAB parameters: first-order indices of the most influential parameters (with index $>1 \%$ ).

$\begin{array}{ccccccc}\operatorname{sinkA}(\text { cap }) & \operatorname{sinkB}(\text { cap }) & \text { sinkA(intern) } & \text { SR(cap) } & \text { SR(intern) } & \text { sinkB(intern) } & \text { internEpdTT } \\ 0.51 & 0.12 & 0.12 & 0.05 & 0.03 & 0.02 & 0.02\end{array}$


Table 3: Estimated parameter values of SUNLAB for four genotypes.

\begin{tabular}{|c|c|c|c|c|}
\hline \multirow{2}{*}{$\begin{array}{c}\text { Parameter } \\
\text { Name }\end{array}$} & \multicolumn{4}{|c|}{ Param. values (with associated standard error) } \\
\hline & Albena & Melody & Heliasol & Prodisol \\
\hline $\operatorname{sinkA}($ blade $)$ & $8.4(0.22)$ & $2.8(0.12)$ & $2(0.1)$ & $4(0.16)$ \\
\hline $\operatorname{sinkA}($ petiole $)$ & $3.4(0.33)$ & $1.5(0.22)$ & $1.5(0.7)$ & $4.3(0.76)$ \\
\hline $\operatorname{sink} \mathrm{A}($ internode $)$ & $2.2(0.12)$ & $3.5(0.05)$ & $2.2(0.07)$ & $3.8(0.08)$ \\
\hline sinkA(capitulum) & $5.6(0.12)$ & $4.3(0.17)$ & $6.5(0.3)$ & $6.5(0.28)$ \\
\hline sinkB(blade) & $14.8(0.4)$ & $2.3(0.16)$ & $2.1(0.18)$ & $3.6(0.26)$ \\
\hline $\operatorname{sinkB}($ petiole $)$ & 16.8 & $4.1(6.4)$ & $2.7(0.76)$ & $4.2(0.5)$ \\
\hline $\operatorname{sinkB}($ internode $)$ & 13.8 & $7.7_{(0.29)}$ & $1.7_{(0.07)}$ & $12.2(0.44)$ \\
\hline $\operatorname{sinkB(capitulum)}$ & $3.4(0.22)$ & $2.5(0.23)$ & $6.1_{(0.44)}$ & $5.8(0.52)$ \\
\hline $\mathrm{SR}$ (petiole) & $0.5(0.04)$ & $0.2(0.03)$ & $0.24_{(0.03)}$ & $0.43(0.04)$ \\
\hline SR(internode) & $1_{(0.06)}$ & $3(0.19)$ & $1.6_{(0.08)}$ & $1.8_{(0.09)}$ \\
\hline $\mathrm{SR}$ (capitulum) & 1000 & $600(126)$ & $350_{(54)}$ & $500(144)$ \\
\hline
\end{tabular}




\section{List of Figures}

1 Water cycle processes as considered in the water budget module of SUNLAB . . . . . . . . . . . . . . . 56

2 Fraction of transpirable soil water FTSW for three datasets "2001", "2002a", "2002b" ................. . . 57

3 Experimental data (dots) and simulation (lines) comparisons of blade dry mass, internode dry mass, and capitulum dry mass for the four genotypes - "Albena", "Melody", "Heliasol", and "Prodisol" - in dataset "2001". . . . . . . . . . . . . . . . 58

$4 \quad$ Sink strength variation based on SUNLAB estimated parameters 59

5 Graphs A to D: experimental data (dots) and simulations (lines) comparisons for the "2001" (blue) and "2002a" (red) conditions of the radiation interception efficiency $R I E(d)$, total blade area $A A(d)$, leaf number $N(d)$, cumulated dry aboveground biomass $C D M(d)$ and biomass compartments (capitulum, blades, petioles, internodes) for the "Melody" genotype. Graph E: biomass compartments of "Prodisol" genotype. Graph F: experimental data (dots) and simulations (lines) comparisons for individual blade biomass of "Heliasol" genotype on different days in dataset "2001" (blue) and "2002a" (red). . . . . . . . . . . . . . . 60

6 Model evaluation for genotype "Albena" using an additional experimental dataset: "2002b" (RMSE: 36.4; coefficient of determination: 0.95) . . . . . . . . . . . . 61

7 Comparison of simulation and field data for individual blade area and biomass of genotype "Melody"; the right graph is the simulation of specific leaf area for the four genotypes . . . . . 62 


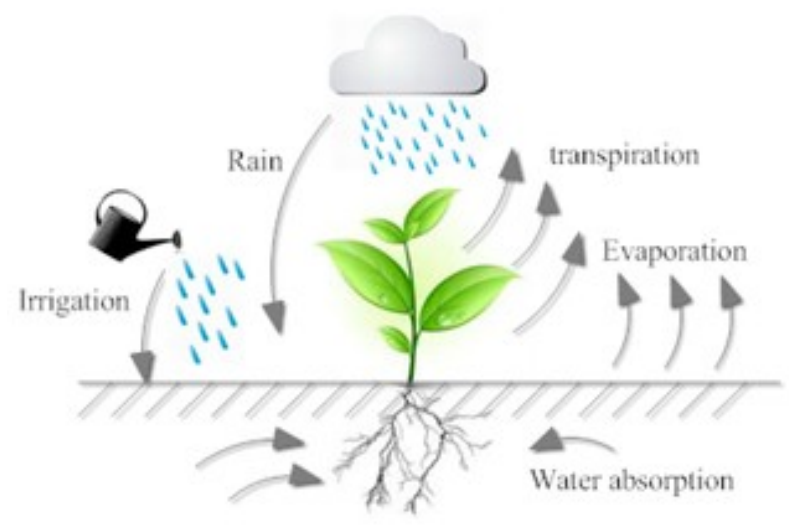

Figure 1: Water cycle processes as considered in the water budget module of SUNLAB 

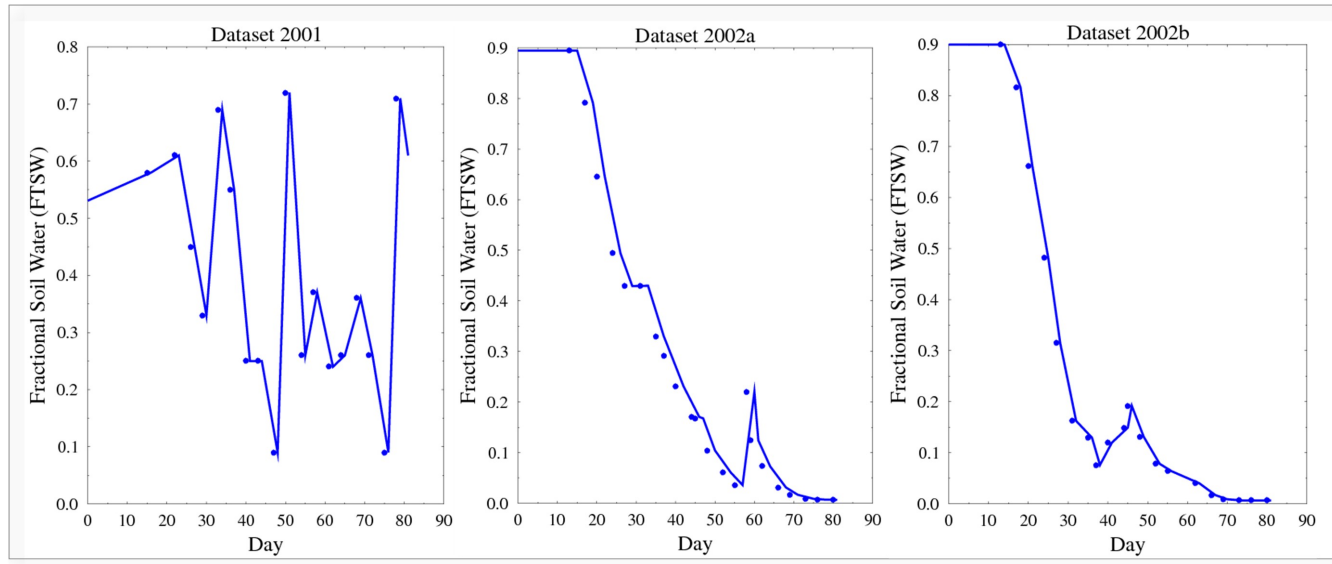

Figure 2: Fraction of transpirable soil water FTSW for three datasets "2001", "2002a", "2002b" 

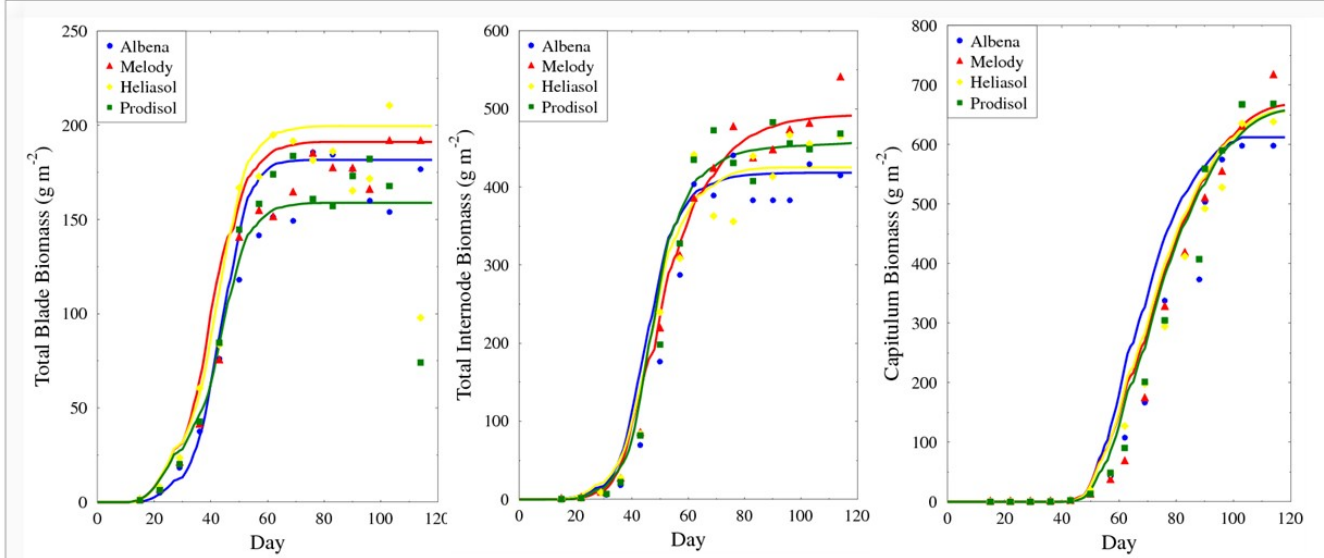

Figure 3: Experimental data (dots) and simulation (lines) comparisons of blade dry mass, internode dry mass, and capitulum dry mass for the four genotypes - "Albena", "Melody", "Heliasol", and "Prodisol" - in dataset "2001". 


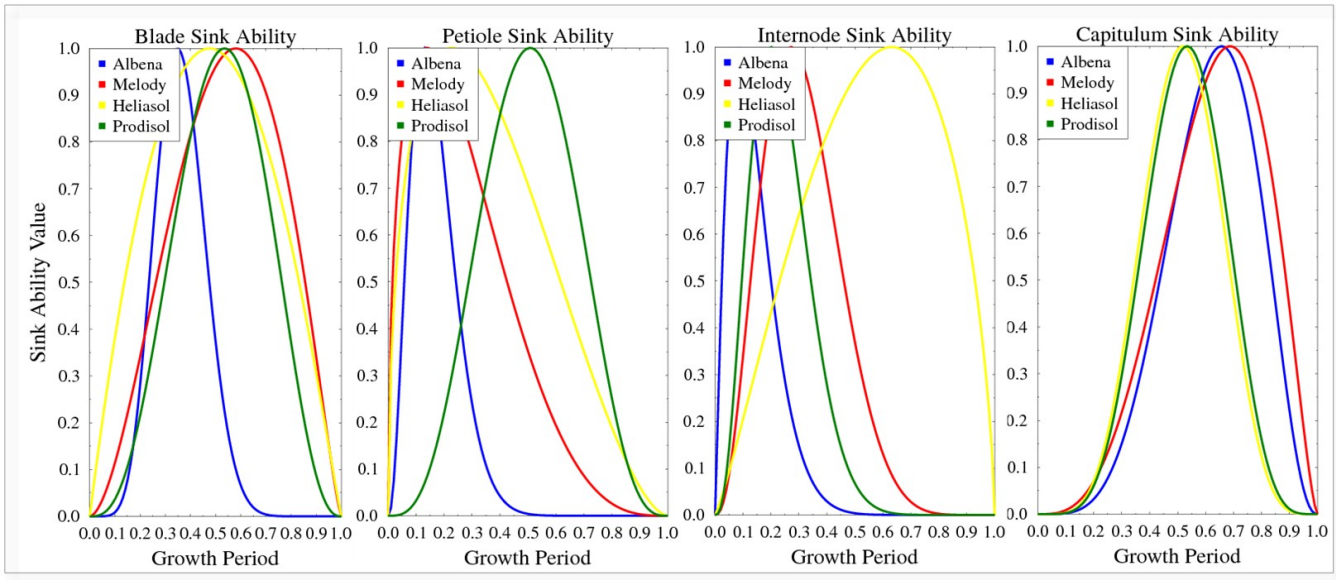

Figure 4: Sink strength variation based on SUNLAB estimated parameters 

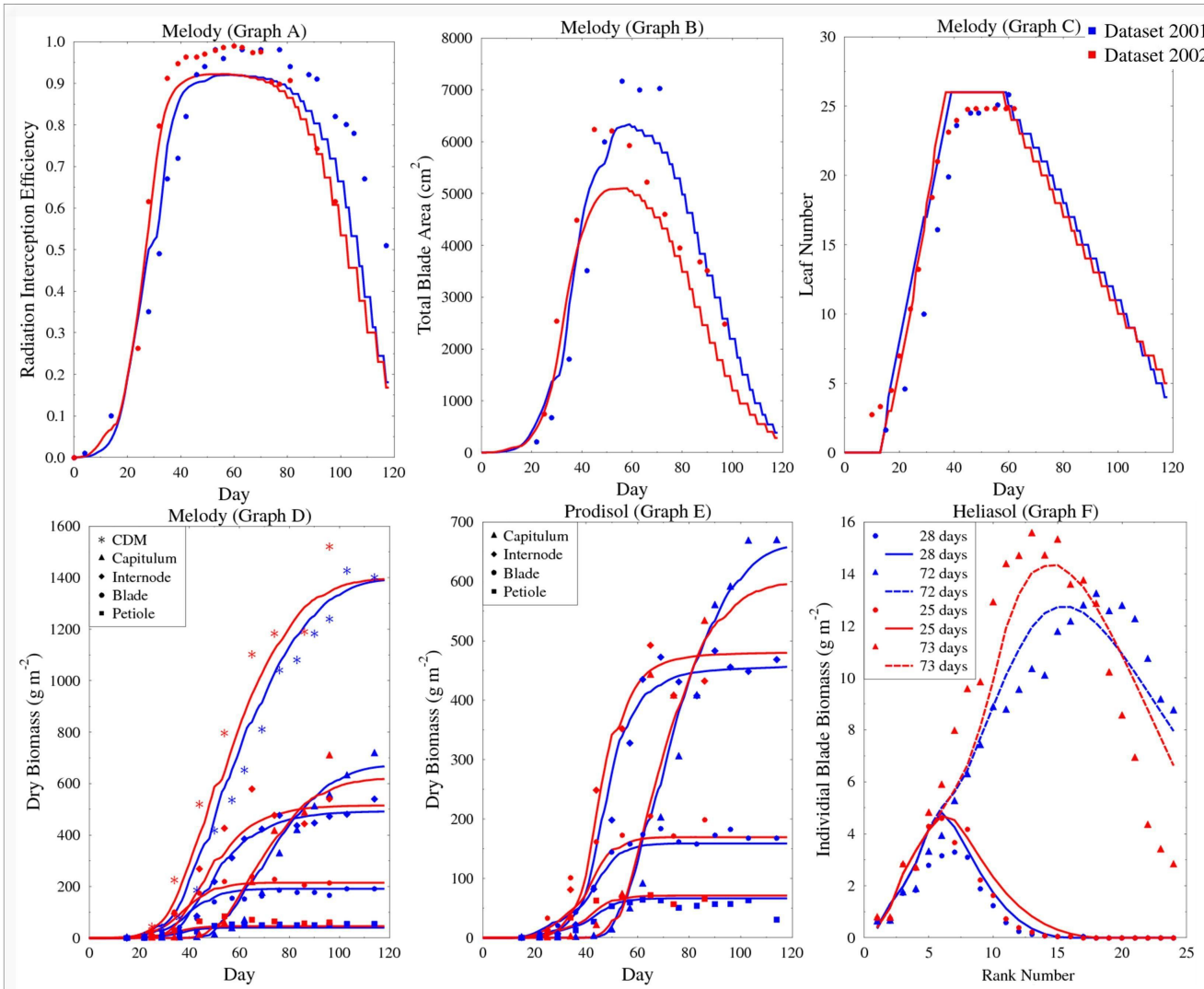

Figure 5: Graphs A to D: experimental data (dots) and simulations (lines) comparisons for the "2001" (blue) and "2002a" (red) conditions of the radiation interception efficiency $R I E(d)$, total blade area $A A(d)$, leaf number $N(d)$, cumulated dry above-ground biomass $C D M(d)$ and biomass compartments (capitulum, blades, petioles, internodes) for the "Melody" genotype. Graph E: biomass compartments of "Prodisol" genotype. Graph F: experimental data (dots) and simulations (lines) comparisons for individual blade biomass of "Heliasol" genotype on different days in dataset "2001" (blue) and "2002a" (red). 

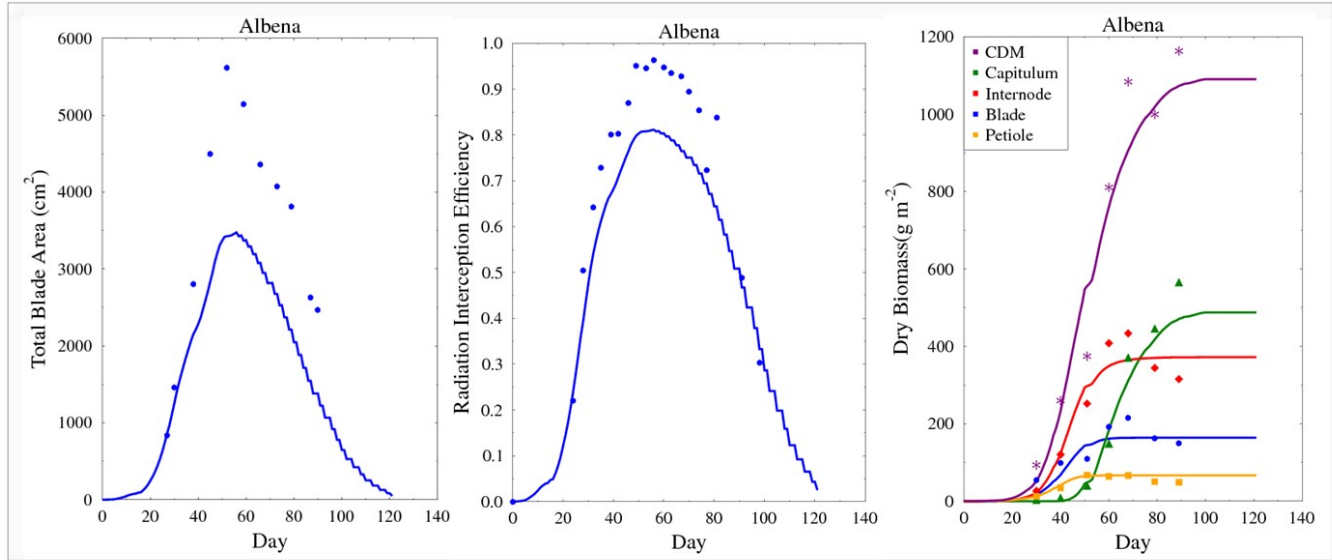

Figure 6: Model evaluation for genotype "Albena" using an additional experimental dataset: "2002b" (RMSE: 36.4; coefficient of determination: 0.95) 

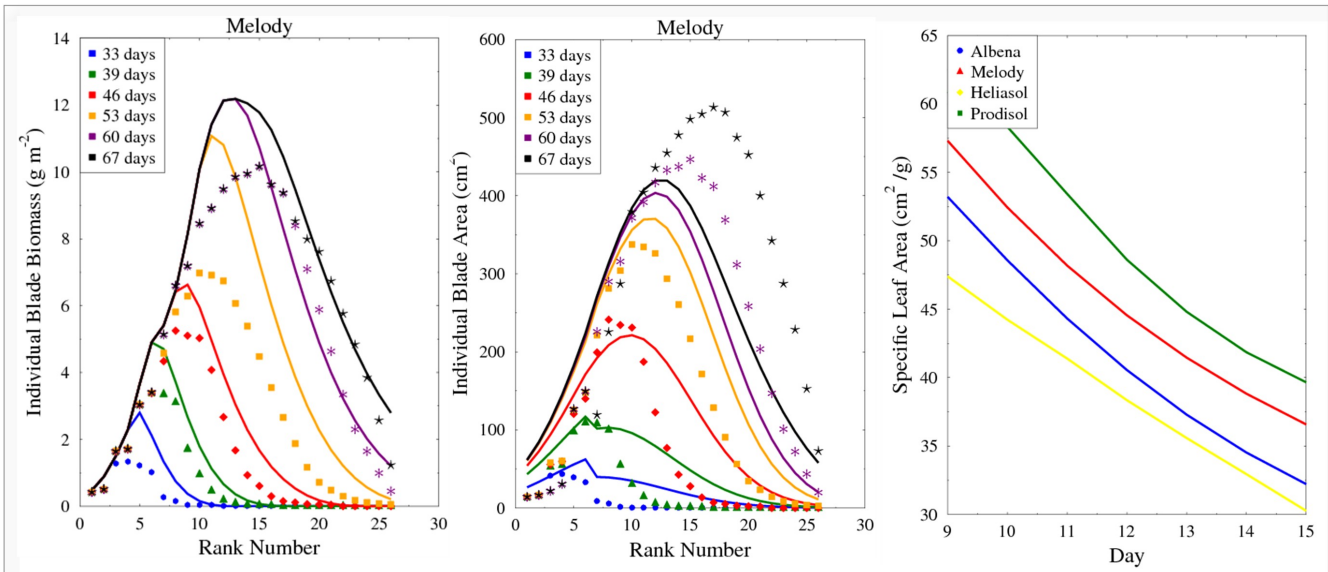

Figure 7: Comparison of simulation and field data for individual blade area and biomass of genotype "Melody"; the right graph is the simulation of specific leaf area for the four genotypes 